\title{
Laparoscopic vertical sleeve gastrectomy, long and short-term impact on weight loss and associated co-morbidities
}

\author{
Leena Khaitan, Brian J. Shea \\ Department of Surgery, University Hospitals Cleveland Medical Center, Cleveland, OH, USA \\ Contributions: (I) Conception and design: All authors; (II) Administrative support: Department of Surgery, University Hospitals Cleveland Medical \\ Center, Cleveland, OH; (III) Provision of study materials or patients: None; (IV) Collection and assembly of data: All authors; (V) Data analysis and \\ interpretation: All authors; (VI) Manuscript writing: All authors; (VII) Final approval of manuscript: All authors. \\ Correspondence to: Brian J. Shea, MD. University Hospitals Cleveland Medical Center, Department of Surgery, 11100 Euclid Ave, Cleveland, OH \\ 44106, USA. Email: Brian.Shea@uhhospitals.org.
}

\begin{abstract}
The laparoscopic vertical sleeve gastrectomy (LVSG) has become the most popular operation for the treatment of morbid obesity in the United States. Being a purely restrictive procedure, the LVSG works to reduce the caloric intake of patients as well as decrease appetite through removal of ghrelin producing cells. Initially developed as the first part of a combined two step restrictive and malabsorptive procedure, the LVSG developed as a standalone procedure when patients lost significant weight with the restrictive portion of the operation alone. Short term outcomes have been promising in terms of weight loss and resolution of comorbid conditions. Long term outcomes are still evolving, but do demonstrate durable weight loss for a significant number of patients. Concerns with the LVSG in the long term revolve around development or worsening of gastroesophageal reflux disease or weight regain. The LVSG has been demonstrated to be a useful tool in the surgical management of morbid obesity.
\end{abstract}

Keywords: Sleeve; outcomes; bariatric; weight loss

Submitted Dec 20, 2019. Accepted for publication Jan 14, 2020.

doi: 10.21037/atm.2020.01.89

View this article at: http://dx.doi.org/10.21037/atm.2020.01.89

\section{Introduction}

Bariatric surgery has been demonstrated to be the most successful approach to long term weight loss in morbidly obese patients. Several previous studies have demonstrated the efficacy of the surgical approach in the treatment of obesity and its related comorbid conditions (1-4). Surgical weight loss procedures can be classified as restrictive or malabsorptive in terms of mechanism of action. There have been several previous attempts to create a durable restrictive weight loss procedure. The vertical banded gastroplasty (VBG) was one such procedure, in which a window through both walls of the proximal stomach was created and a stapler fired towards the angle of His to create a small pouch. A polypropylene mesh collar could then be placed around the pouch to keep the volume constant (5). This procedure was abandoned due to its inferiority in terms of weight loss when compared to the RYGB (6-8), and a high incidence of reoperation $(9,10)$. The VBG gave way to the laparoscopic adjustable gastric band (LAGB), which was placed around the proximal portion of the stomach to create a feeling of early satiety. The band had the advantage of being adjustable, allowing for steady increases in band volume over time. However complications like slippage or erosion (11) as well as inferior weight loss (12) led to decreased utilization of this procedure. As a result, the current most common procedure related to the LAGB is their removal (13).

The failure of these procedures to provide a safe restrictive option opened the door for the development of the laparoscopic vertical sleeve gastrectomy (LVSG). Initially, the LVSG was used as the first part of a duodenal switch procedure, providing a degree of restriction with the small intestinal bypass (14). The LVSG had been utilized in super obese patients to allow for initial weight loss prior to 
completion of the small intestinal bypass. It was observed that these patients would lose a significant amount of excess weight and improve comorbidities even prior to completion of the small intestinal bypass, leading to its development as a standalone procedure (15). The LSVG is now approved for surgical management of obesity in patients with body mass index $(\mathrm{BMI})>35 \mathrm{~kg} / \mathrm{m}^{2}$ with one obesity related comorbidity or for patients with $\mathrm{BMI}>40 \mathrm{~kg} / \mathrm{m}^{2}$. The popularity of the LVSG has been steadily increasing over the last several years due to its favorable safety profile and low complexity, and is now the most commonly utilized bariatric operation in the United States, with the LSVG occupying $59.38 \%$ of all bariatric operations performed in 2017 (16).

In the following manuscript, technical considerations for the LSVG will be discussed, as well as short and long term outcomes in terms of weight loss and resolution of obesity related comorbid conditions. Long term concerns with this procedure will also be discussed, including long term weight loss and resolution of comorbidities, gastroesophageal reflux and weight regain.

\section{Technical considerations}

In this section, technical considerations for the LVSG will be discussed in their relation to short-term outcomes. Technical variables involved in this process are the size of the remaining stomach as defined by the size of the sizing device used as the guide for the stapler, the distance from the pylorus where the staple line starts, and staple line reinforcement.

\section{Bougie size}

During creation of a sleeve-like stomach, it has been recommended to use a sizing device to ensure a consistent sleeve staple line and adequate volume. A bougie or other sizing device can be used for this purpose, placed transorally and fed along the lesser curvature of the stomach. One would assume that a smaller bougie size would lead to a smaller stomach, resulting in increased restriction and ultimately increased weight loss. This principle was suggested in early data from a series of 540 patients by Sánchez-Santos et al. (17) in which smaller bougie size (32-38 French) led to superior weight loss when compared to use of a larger bougie and did not increase complications.

More recent studies incorporating more patients appear to refute that assertion. Not only does a smaller bougie size not increase weight loss, but it also leads to increase risk of leak. A study by Yuval et al. (18) of 4,999 patients examined bougie use in patients undergoing LVSG. Bougie size less than 40 French led to an excess weight loss of $60.7 \%$, while patients with bougie size of greater than 40 French had an excess weight loss of $69.2 \%$, the difference between the two not achieving statistical significance. Additionally, leak rates with the smaller bougie size was noted to be $2.67 \%$ compared to $0.92 \%$, which was significant. Another larger systematic review and meta-analysis by Parikh et al. (19) of 9,991 patients who underwent LVSG had similar findings. This study demonstrated no difference in excess weight loss at three years with use of a bougie smaller than 40 French compared with those over 40 French, and demonstrated a higher leak rate with the use of a smaller bougie. With this information, in order to provide adequate weight loss without increasing risk of leak, a bougie size of around 40 French appears to be safe.

\section{Distance from pylorus}

When creating a sleeve, an important technical consideration becomes how long to make the staple line. The staple line has a defined endpoint at the angle of his, but variability exists with where to start the staple line in relation to the pylorus. If the staple line is initiated close to the pylorus, more stomach can be removed, creating restriction over a longer distance.

Previous studies have sought to determine the effect of antral resection on weight loss. Two prospective, randomized trials in Egypt were performed to determine if degree of antral resection had an impact on complications or weight loss after LVSG. Abdallah et al. (20) randomized 105 patients to undergo a LVSG with staple line starting either two centimeters from the pylorus (group 1) or $6 \mathrm{~cm}$ from the pylorus (group 2). The results of this study demonstrated improved long-term weight loss with greater antral resection ( $71.8 \%$ vs. $61 \%$ at 24 months). ElGeidie et al. (21) randomized 113 patients to undergo either an antral resecting or an antral preserving LVSG, with measurements from pylorus the same as the aforementioned study. Contrary to the results of the other trial, there were no differences found in terms of weight loss or complications between the two approaches.

More recent studies have also examined the impact of distance from the pylorus on outcomes.

An examination of the ACS-MBSAQIP database of 170,751 patients found that patients who underwent LVSG 
in which the staple line was more than $4 \mathrm{~cm}$ from the pylorus were less likely to be readmitted following their operation and less likely to be treated for dehydration when compared to patients whose staple lines started less than $4 \mathrm{~cm}$ from the pylorus (22). A recent study examined the impact of sleeve shape on weight loss, with sleeves that have staple lines close to the pylorus creating more of a tubular shape, while sleeves with staple lines starting further away from the pylorus create a "lower pouch" shape. This study of 210 patients found that patients that had the lower pouch type staple line demonstrated a trend toward decreased reflux and improved weight loss when compared to other shapes including the tubular sleeve (23).

Data with larger sample sizes appear to favor starting the staple line approximately $4-6 \mathrm{~cm}$ from the pylorus. A greater antral resection does not appear to add benefit in terms of weight loss. Starting the staple line closer to the pylorus also may narrow the sleeve at the incisura angularis, which is the most common site of stricture formation after LVSG (24), which may have long term implications for patients.

\section{Staple line reinforcement}

The LVSG creates a long staple line up the greater curvature of the stomach, and creates an area that can be prone to bleeding. To combat this common complication, many surgeons favor reinforcing the staple line. Options include reinforcement by oversewing or the use of synthetic or bioabsorbable material.

Two analyses of the MBSAQIP database aimed to identify the role of staple line reinforcement in the LVSG, and its impact on bleeding and leak rate. Both studies found that any form of reinforcement is better than no reinforcement in terms of bleeding rates. An examination of the 2016 data from the database demonstrated a bleeding rate of $0.6 \%$ in the no reinforcement group versus $0.4 \%$ (OR 0.7, $\mathrm{P}=0.007$ ) in the staple line reinforcement group and $0.5 \%(\mathrm{OR} 0.93, \mathrm{P}=0.7)$ in the oversewing group. If both techniques were used for reinforcement, the rate fell to $0.3 \%(\mathrm{OR} 0.52, \mathrm{P}=0.002)$ (25). A study of the compiled 2015 and 2016 data showed similar results, favoring staple line reinforcement to lower bleeding rates (26).

While several studies have demonstrated the benefit of staple line reinforcement for the purposes of reducing bleeding, the results on its effect on leak rates have been mixed. Furthermore the above MBSAQIP analyses demonstrated no difference in leak rates between any of the approaches to staple line reinforcement (no reinforcement, oversewing or use of reinforcement), two systematic reviews have demonstrated certain staple line reinforcement to reduce leak rates. A systematic review of 88 studies including 8,920 patients demonstrated significantly lower leak rates with use of an absorbable polymer membrane (APM), when compared to no reinforcement or reinforcement with bovine pericardial membrane (BPM) (1.09\% vs. $2.6 \%$ vs. $3.3 \%$ ) (27). A larger systematic review incorporating 40,653 patients demonstrated similar results, with reinforcement using APM achieving lower leak rates compared to no reinforcement or BPM reinforcement (28).

A more recent analysis by Berger et al. (29) of the MBSAQIP contradicts these findings. In their analysis of 189,477 patients who underwent surgery between 2012 and 2014, while staple line reinforcement decreased bleeding rates, they actually found that reinforcement increased risk of leak $(0.96 \%$ with reinforcement, $0.65 \%$ without, OR 1.20). While the increased leak rate found in this study is poorly understood, one possible explanation is the sandwiching of reinforcement layers in overlap zones, creating areas of potential weakness and ischemia. The authors utilize staple line reinforcement routinely.

\section{Short term outcomes}

The LVSG has been demonstrated to be a safe and well tolerated operation. Earlier studies demonstrated bariatric surgery to be a risky endeavor, especially in elderly patients. A 2005 study of Washington Medicare beneficiaries demonstrated a higher than expected early mortality rate (30), leading to a national coverage decision restricting reimbursement for bariatric surgery (31). Since then, with time and experience, bariatric surgery has been demonstrated to be safe and effective. A follow up study after the national coverage decision demonstrated improved outcomes amongst Medicare beneficiaries (32).

Further data has reinforced the safety of bariatric surgery. The Longitudinal Assessment of Bariatric Surgery Consortium published a study demonstrating a mortality rate of $0.3 \%$ and a major adverse event rate of $4.3 \%$ within 30 days in their patient population of 4,776 patients (33). When referring to LVSG operations specifically, other studies have aimed to judge their safety when compared to the gold standard of bariatric operations: the Rouxen-Y gastric bypass (RYGB). An analysis if the NSQIP database analyzed 24,117 patients who underwent LVSG or bypass for morbid obesity. When compared with RYGB, 
patients who had a LVSG had a shorter operative time (101 vs. 130 minutes), and lower rates of blood loss requiring transfusion $(0.6 \%$ vs. $1.5 \%)$, deep wound infection $(0.06 \%$ vs. $0.2 \%)$, serious morbidity rate $(3.8 \%$ vs. $5.8 \%)$, and 30 -day reoperation rate (1.6\% vs. $2.5 \%)$ (34). An analysis of the 2015 MBSAQIP database of 134,142 patients demonstrated a lower mortality rate $(0.1 \%$ vs. $0.2 \%)$, morbidity rate $(5.8 \%$ vs. $11.7 \%)$, and leak rate $(0.8 \%$ vs. $1.6 \%)$ in patients undergoing LVSG when compared to RYGB (35).

LVSG can have a very significant impact on weight and resolution of obesity related comorbidities in the short term. The volume restriction with the LVSG reduces the number of calories a patient can eat at a time, resulting in decreased overall caloric intake and weight loss. Additionally, the LVSG also removes the Ghrelin producing cells in the stomach, decreasing appetite (36). Studies of short-term outcomes have demonstrated significant weight loss. A systematic review that included 5,218 patients demonstrated an excess weight loss of $67.3 \%$, resulting in a significant $\mathrm{BMI}$ reduction. The procedure also had a significant impact on comorbidities, with $81.9 \%$ of patients with diabetes, $66.5 \%$ of patients with hypertension, and $64.1 \%$ of patients with dyslipidemia experiencing remission at one year (37).

A direct comparison to the LRYGB was performed by randomizing patients to undergo RYGB or LVSG. The SLEEVEPASS trial enrolled 240 patients to undergo LVSG or RYGB. At six months, patients in the sleeve group when compared to patients in the bypass group had similar rates of excess weight loss (49.2\% vs. $52.9 \%$ ), resolution or improvement in Diabetes (84.3\% vs. 93.3\%), hypertension (76.8\% vs. $81.9 \%)$ and hypercholesterolemia (64.1\% vs. $69.0 \%)(38)$.

One of the most feared short-term complications of the LVSG is a staple line leak. A leak after LVSG may occur due to decreased blood supply to part of the staple line, resulting in ischemia and an eventual leak. The most common location for a leak is in the proximal third of the stomach, as demonstrated by a systematic review of 4,889 which demonstrated that $89 \%$ of leaks were in this location (39). This may result from alteration in blood flow in this area that a LVSG can create. A study of sleeve gastrectomies of cadaveric trunks demonstrated that branches to the possible part of the gastric tube from the left gastric artery can be damaged by the LVSG, making this area particularly vulnerable to ischemia (40). Patients particularly vulnerable to this complication include those who are oxygen dependent, or have obstructive sleep apnea, hypoalbuminemia, hypertension or diabetes, as demonstrated by an analysis of the 2015 MBSAQIP database (41).

\section{Long term outcomes}

Long term data for the LVSG is starting to become available as the utilization of the LVSG as a standalone bariatric procedure has increased over the past several years. With past restrictive procedures, there were long-term difficulties with durability of weight loss. Several studies with long term follow up have demonstrated the durability of this operation.

Much of the data regarding long term outcomes from LVSG comes from institutionally reported data. A systematic review examining weight loss in patients that underwent LVSG incorporated 15 studies and 432 patients. Weight loss outcomes were measured for patients with at least five years of follow up, and found that these patients had a mean excess weight loss of $59.3 \%$ (42). A larger systematic review of 20 studies incorporated 1,626 patients with at least five years of follow up demonstrated durable weight loss and improvement of obesity related comorbid conditions. Patients with that length of follow up demonstrated $58.4 \%$ excess weight loss. Patients that followed up for longer intervals (up to 11 years) appeared to maintain that weight loss through follow up. Patients also saw improvement or resolution in diabetes $(77.8 \%)$, hypertension (68\%) dyslipidemia (65.9\%), obstructive sleep apnea $(75.8 \%)$, gastroesophageal reflux disease $(430.6 \%)$, and degenerative joint disease (55.7\%) (43).

Two randomized controlled trials have compared outcomes of the LVSG and the RYGB. The SLEEVEPASS trial was a study of 240 patients randomized to undergo either LVSG or RYGB in Finland. Five-year data of that trial has recently been reported, and demonstrated mean excess weight loss of $49 \%$ in sleeve patients compared to $57 \%$ in bypass patients, a difference which was not statistically significant. Patients also had similar rates of complete or partial remission of comorbid conditions including diabetes and hyperlipidemia, and patients in the bypass group were more likely to experience complete or partial remission of hypertension (44). Similarly, the SMBOSS trial of 217 Swiss patients randomized to undergo LVSG or RYGB demonstrated similar weight loss outcomes between the two groups, as well as similar rates of resolution of Diabetes and hyperlipidemia (45).

Long-term outcomes were compared in a systematic review and meta-analysis incorporating 14 studies and 5,264 patients who underwent LVSG or LRYGB. A slight 
weight loss benefit in terms of long-term weight loss was demonstrated for patients that underwent LRYGB, although there was a substantial benefit in terms of long-term weight loss for those who underwent LVSG as well. Resolution of comorbid conditions was similar between the two groups (46).

Although long-term data for outcomes from LVSG is still being developed, the available data from studies with longterm follow up demonstrate the promise of this procedure. Compared with the gold standard bariatric operation that is the RYGB, the LVSG offers weight loss close to that of the RYGB, with similar rates of resolution or improvement in obesity related comorbid conditions. The issue with previous purely restrictive operations, however, was the reintervention rate after the initial operation. This is an issue that is also starting to develop with the LVSG.

One major issue for patients that undergo LVSG is the potential worsening of existing gastroesophageal reflux disease or the development of de novo reflux. A study of 20 post-operative LVSG patients that underwent $\mathrm{pH}$ monitoring demonstrated a significant increase in acid exposure when compared to pre-operative values, indicating the effect a LVSG can have on reflux. Interestingly, the increased acid exposure had different effects of patient's symptoms. Fourteen of the twenty patients in this study had pre-operative reflux. In those patients, after the operation six reported improved symptoms, six reported worsening symptoms, and two had no change. Of the 6 patients without reflux prior to the operation, four complained of de novo reflux symptoms (47). This increased acid exposure can have a potential impact on the esophagus, as a study of 110 patients who underwent LVSG underwent subsequent follow up endoscopy and found a $17.2 \%$ incidence of new Barrett's esophagus (48). While the above studies did not demonstrate a correlation with acid exposure and reflux symptoms, symptomatic reflux remains a common indication for revision operation after LVSG.

As long-term data regarding the LVSG continues to be developed, revisional surgery for patients with a purely restrictive procedure with the LVSG continues to be a concern. A meta-analysis encompassing 2,280 LVSG patients with at least 7 years of follow up demonstrated a weight recidivism rate of $27.8 \%$, with a revisional operation rate of $19.9 \%$ (49). An analysis of Statewide Planning and Research Cooperative System in New York State of 40,994 bariatric procedures found a lower revision rate than the previous study in this population of $9.8 \%$. However, this rate was still twice the revision rate of a RYGB (4.9\%) (50). Most common indications for revision surgery include gastroesophageal reflux not manageable with medical therapy and weight regain. Options for revision include conversion to RYGB or a biliopancreatic diversion. These data demonstrate some concerns with the LVSG similar to that of other purely restrictive operations.

\section{Conclusions}

The LVSG has become the most popular bariatric operation in the United States for the treatment of morbid obesity due to its technical simplicity and palatability to patients. The weight loss associated with the LVSG has been demonstrated to be substantial, and many patients continue to keep the weight off in the long term. The data available also demonstrate improvement in obesity related comorbid conditions, though long-term data is still being developed. It is important to remember that the LVSG was initially developed as the restrictive portion of a malabsorptive procedure, and previous attempts at a purely restrictive procedure have been abandoned due to insufficient weight loss or high revision surgery rate. While the initial data with the LVSG are promising, the development or worsening of reflux as well as the revisional surgery rate should be kept in mind when discussing surgical options with patients.

\section{Acknowledgments}

Acknowledgement to Dr. Memon for including our work in his dedicated issue of $A T M$.

Funding: None.

\section{Footnote}

Provenance and Peer Review: This article was commissioned by the Guest Editor (Muhammed Ashraf Memon) for the focused issue "Bariatric Surgery" published in Annals of Translational Medicine. The article was sent for external peer review organized by the Guest Editor and the editorial office.

Conflicts of Interest: The focused issue "Bariatric Surgery" was commissioned by the editorial office without any funding or sponsorship. The authors have no conflicts of interest to declare.

Etbical Statement: The authors are accountable for all aspects of the work in ensuring that questions related to the accuracy or integrity of any part of the work are 
appropriately investigated and resolved.

Open Access Statement: This is an Open Access article distributed in accordance with the Creative Commons Attribution-NonCommercial-NoDerivs 4.0 International License (CC BY-NC-ND 4.0), which permits the noncommercial replication and distribution of the article with the strict proviso that no changes or edits are made and the original work is properly cited (including links to both the formal publication through the relevant DOI and the license). See: https://creativecommons.org/licenses/by-nc-nd/4.0/.

\section{References}

1. Mingrone G, Panunzi S, De Gaetano A, et al. Bariatric Surgery versus Conventional Medical Therapy for Type 2 Diabetes. N Engl J Med 2012;366:1577-85.

2. Schauer PR, Kashyap SR, Wolski K, et al. Bariatric Surgery versus Intensive Medical Therapy in Obese Patients with Diabetes. N Engl J Med 2012;366:1567-76.

3. Jakobsen GS, Småstuen MC, Sanbu R, et al. Association of Bariatric Surgery vs Medical Obesity Treatment With Long-term Medical Complications and Obesity-Related Comorbidities. JAMA 2018;319:291-301.

4. Ribaric G, Buchwald JN, McGlennon TW. Diabetes and Weight in Comparative Studies of Bariatric Surgery vs Conventional Medical Therapy: A Systematic Review and Meta-Analysis. Obes Surg 2014;24:437-55.

5. Mason EE. Vertical Banded Gastroplasty for Obesity. Arch Surg 1982;117:701-6.

6. Olbers T, Fagevik-Olsén M, Maleckas A, et al. Randomized clinical trial of laparoscopic Roux-en-Y gastric bypass versus laparoscopic vertical banded gastroplasty for obesity. Br J Surg 2005;92:557-62.

7. Brolin RE, Robertson LB, Kenler HA, et al. Weight loss and dietary intake after vertical banded gastroplasty and Roux-en-Y gastric bypass. Ann Surg 1994;220:782-90.

8. Balsiger BM, Poggio JL, Mai J, et al. Ten and more years after vertical banded gastroplasty as primary operation for morbid obesity. J Gastrointest Surg 2000;4:598-605.

9. Marsk R, Jonas E, Gartzios H, et al. High revision rates after laparoscopic vertical banded gastroplasty. Surg Obes Relat Dis 2009;5:94-8.

10. Miller K, Pump A, Hell E. Vertical banded gastroplasty versus adjustable gastric banding: prospective long-term follow-up study. Surg Obes Relat Dis 2007;3:84-90.

11. Singhal R, Bryant C, Kitchen M, et al. Band slippage and erosion after laparoscopic gastric banding: a meta- analysis. Surg Endosc 2010;24:2980-6.

12. Garb J, Welch G, Zagarins S, et al. Bariatric Surgery for the Treatment of Morbid Obesity: A Meta-analysis of Weight Loss Outcomes for Laparoscopic Adjustable Gastric Banding and Laparoscopic Gastric Bypass. Obes Surg 2009;19:1447-55.

13. Koh CY, Inaba CS, Sujatha-Bhaskar S, et al. Laparoscopic Adjustable Gastric Band Explantation and Implantation at Academic Centers. J Am Coll Surg 2017;225:532-7.

14. Brethauer SA. Sleeve Gastrectomy. Surg Clin N Am 2011;91:1265-79.

15. Cottam D, Qureshi FG, Mattar SG, et al. Laparoscopic sleeve gastrectomy as an initial weight loss procedure for high-risk patients with morbid obesity. Surg Endosc 2006;20:859-63.

16. American Society for Metabolic and Bariatric Surgery. (June, 2018). Estimate of Bariatric Surgery Numbers, 2011-2017. Available online: https://asmbs.org/resources/ estimate-of-bariatric-surgery-numbers

17. Sánchez-Santos R, Masdevall C, Baltasar A, et al. Shortand mid-term outcomes of sleeve gastrectomy for morbid obesity: the experience of the Spanish National Registry. Obes Surg 2009;19:1203-10.

18. Yuval JB, Mintz Y, Cohen MJ, et al. The Effects of Bougie Caliber on Leaks and Excess Weight Loss Following Laparoscopic Sleeve Gastrectomy. Is There and Ideal Bougie Size? Obes Surg 2013;23:1685-91.

19. Parikh M, Issa R, McCrillis A, et al. Surgical Strategies That May Decrease Leak After Laparoscopic Sleeve Gastrectomy: A Systematic Review and Meta-Analysis of 9991 cases. Ann Surg 2013;257:231-7.

20. Abdallah E, El Nakeeb A, Yousef T, at al. Impact of Extent of Antral Resection on Surgical Outcomes of Sleeve Gastrectomy for Morbid Obesity (A Prospective Randomized Study). Obes Surg 2014;24:1587-94.

21. ElGeidie A, ElHemaly M, Hamdy E, et al. the effect of residual gastric antrum size on the outcome of laparoscopic sleeve gastrectomy: a prospective randomized trial. Surg Obes Relat Dis 2015;11:997-1003.

22. Haskins IN, Jackson HT, Graham AE, et al. The effect of bougie size and distance from pylorus on dehydration after laparoscopic sleeve gastrectomy: an analysis of the ACS-MBSAQIP database. Surg Obes Relat Dis 2019;15:1656-61.

23. Alhaj Saleh A, Janik MR, Mustafa RR, et al. Does Sleeve Shape Make a Difference in Outcomes? Obes Surg 2018;28:1731-7.

24. Sarkhosh K, Birch DW, Sharma A, et al. Complications 
associated with laparoscopic sleeve gastrectomy for morbid obesity: a surgeon's guide. Can J Surg 2013;56:347-52.

25. El Chaar M, Stoltzfus J. Assessment of Sleeve Gastrectomy Surgical Technique: First Look at 30-day Outcomes Based on the MBSAQIP Database. J Am Coll Surg 2018;227:564-72.

26. Demeusy A, Sill A, Averbach A. Current role of staple line reinforcement in 30-day outcomes of primary laparoscopic sleeve gastrectomy: an analysis of MBSAQIP Data, 2015-2016 PUF. Surg Obes Relat Dis 2018;14:1454-61.

27. Gagner M, Buchwald JN. Comparison of laparoscopic sleeve gastrectomy leak rates in four staple-line reinforcement options: a systematic review. Surg Obes Relat Dis 2014;10:713-23.

28. Gagner M, Kemmeter P. Comparison of laparoscopic sleeve gastrectomy leak rates in five staple-line reinforcement options: a systematic review. Surg Endosc 2020;34:396-407.

29. Berger ER, Clements RH, Morton JM, et al. The Impact of Different Surgical Techniques on Outcomes in Laparoscopic Sleeve Gastrectomies: The First Report from the Metabolic and Bariatric Surgery Accreditation Quality Improvement Program (MBSAQIP). Ann Surg 2016;264:464-73

30. Flum DR, Salem L, Broeckel Elrod JA, et al. Early Mortality Among Medicare Beneficiaries Undergoing Bariatric Surgical Procedures. JAMA 2005;294:1903-8.

31. Flum DR, Kwon S, MacLeod K, et al. The use, safety and cost of bariatric surgery before and after Medicare's national coverage decision. Ann Surg 2011;254:860-5.

32. Nguyen NT, Hohmann S, Slone J, et al. Improved bariatric surgery outcomes for Medicare beneficiaries after implementation of the medicare national coverage determination. Arch Surg 2010;145:72-8.

33. Longitudinal Assessment of Bariatric Surgery (LABS) Consortium, Flum DR, Belle SH, et al. Perioperative Safety in the Longitudinal Assessment of Bariatric Surgery. N Engl J Med 2009;361:445-54.

34. Young MT, Gebhart A, Phelan MJ, et al. Use and Outcomes of Laparoscopic Sleeve Gastrectomy vs Laparoscopic Gastric Bypass: Analysis of the American College of Surgeons NSQIP. J Am Coll Surg 2015;220:880-5.

35. Kumar SB, Hamilton BC, Wood SG, et al. Is laparoscopic sleeve gastrectomy safer than laparoscopic gastric bypass? A comparison of 30-day complications using the MBSAQIP data registry. Surg Obes Relat Dis 2018;14:264-9.

36. Langer FB, Reza Hoda MA, Bohdjalian A, et al. Sleeve Gastrectomy and Gastric Banding: Effects on Plasma Ghrelin Levels. Obes Surg 2005;15:1024-9.

37. Emile SH, Elfeki H, Elalfy K, et al. Laparoscopic Sleeve Gastrectomy Then and Now: An Updated Systematic Review of the Progress and Short-term Outcomes Over the Last 5 Years. Surg Laparosc Endosc Percutan Tech 2017;27:307-17.

38. Helmiö M, Victorzon M, Ovaska J, et al. Comparison of short-term outcome of laparoscopic sleeve gastrectomy and gastric bypass in the treatment of morbid obesity: A prospective randomized controlled multicenter SLEEVEPASS study with 6-month follow-up. Scand J Surg 2014;103:175-81.

39. Aurora AR, Khaitan L, Saber A. Sleeve gastrectomy and the risk of leak: A systematic analysis of 4,888 patients. Surg Endosc 2012;26:1509-15.

40. Perez M, Brunaud L, Kedaifa S, et al. Does Anatomy Explain the Origin of a Leak after Sleeve Gastrectomy? Obes Surg 2014;24:1717-23.

41. Alizadeh RF, Li S, Inaba C, et al. Risk Factors for Gastrointestinal Leak after Bariatric Surgery: MBSAQIP Analysis. J Am Coll Surg 2018;227:135-41.

42. Diamantis T, Apostolou KG, Alexandrou A, et al. Review of long-term weight loss results after laparoscopic sleeve gastrectomy. Surg Obes Relat Dis 2014;10:177-83.

43. Juodeikis $\check{Z}$, Brimas G. Long-term results after sleeve gastrectomy: A systematic review. Surg Obes Relat Dis 2017;13:693-9.

44. Salminen P, Hemliö M, Ovaska J, et al. Effect of Laparoscopic Sleeve Gastrectomy vs Laparoscopic Rouxen-Y Gastric Bypass on Weight Loss at 5 Years Among Patients with Morbid Obesity: The SLEEVEPASS Randomized Clinical Trial. JAMA 2018;319:241-54.

45. Peterli R, Wolnerhanssen BK, Peters T, et al. Effect of Laparoscopic Sleeve Gastrectomy vs Laparoscopic Rouxen-Y Gastric Bypass on Weight Loss in Patients with Morbid Obesity: The SM-BOSS Randomized Clinical Trial. JAMA 2018;319:255-65.

46. Shoar S, Saber AA. Long-term and midterm outcomes of laparoscopic sleeve gastrectomy versus Rouxen-Y gastric bypass: a systematic review and metaanalysis of comparative studies. Surg Obes Relat Dis 2017;13:170-80.

47. Burgerhart JS, Schotborgh CAI, Schoon EJ, et al. Effect of Sleeve Gastrectomy on Gastroesophageal Reflux. Obes 
Surg 2014;24:1436-41.

48. Genco A, Soricelli E, Casella G, et al. Gastroesophageal reflux disease and Barrett's esophagus after laparoscopic sleeve gastrectomy: a possible, underestimated long-term complication. Surg Obes Relat Dis 2017;13:568-74.

49. Clapp B, Wynn M, Martyn C, et al. Long term (7 or more years) outcomes of the sleeve gastrectomy: a metaanalysis. Surg Obes Relat Dis 2018;14:741-7.

50. Altieri MS, Yang J, Nie L, et al. rate of revisions or conversion after bariatric surgery over 10 years in the state of New York. Surg Obes Relat Dis 2018;14:500-7.

Cite this article as: Khaitan L, Shea BJ. Laparoscopic vertical sleeve gastrectomy, long and short-term impact on weight loss and associated co-morbidities. Ann Transl Med 2020;8(Suppl 1):S5. doi: 10.21037/atm.2020.01.89 